\title{
Angiography suite concept for an interdisciplinary centre for cardiovascular interventions
}

\author{
Ulf K. M. Teichgräber • Christian Wintzer • \\ Bernd Hamm
}

Received: 24 April 2010 / Revised: 25 June 2010 / Accepted: 6 July 2010 /Published online: 29 July 2010

(C) European Society of Radiology 2010

\begin{abstract}
A permanently mounted angiography suite in an operating room (OR) is considered to be a hybrid OR. However, regular use for angiographic interventions is restricted with this setup. We introduce an alternative use of space for the efficient utilisation of an angiographic suite outside the surgical unit. This concept includes three scenarios that describe a modification of the catheter suite according to the specific clinical demands by adapting the workflow.
\end{abstract}

Keywords Interventional radiology · Hybrid OR ·

Angiographic suite $\cdot$ Utilisation concepts · Workflow

\section{Introduction}

Advancements in minimally invasive image-guided techniques have underpinned the development of abnormalities of the aorta formerly treated with open vessel surgery that are now being treated with transfemoral endovascular aneurysm repair (EVAR) applying stent grafts [1]. Both interventional radiologists and vascular surgeons practice these endovascular implantation techniques [2-5]. To accommodate these new endovascular techniques, a merging of the OR and the angiographic suite is desirable.

The implementation of such a hybrid OR within the surgical unit means that an angiography system is permanently installed in the OR. Thus, open vascular

U. K. M. Teichgräber $(\bowtie) \cdot$ B. Hamm

Department of Radiology, Charité Universtätsmedizin Berlin,

Charitéplatz 1,

10117 Berlin, Germany

e-mail: ulf.teichgraeber@charite.de

C. Wintzer

Department of Surgery, Charité Universtätsmedizin Berlin,

Charitéplatz 1,

10117 Berlin, Germany surgeries can be assisted by an intra-procedural angiographic examination, while a primarily endovascular stent-graft therapy via the femoral arteries remains an alternative option [6]. Hybrid ORs have been implemented in various cardiovascular centres, in which percutaneous and endovascular heart valve implantations, combined interventions in coronary arteries and aortic stent-graft implantations are performed by an interdisciplinary team of physicians as part of the clinical routine [7-9]. A hybrid OR requires a suitable OR table for primarily surgical purposes. Such an OR table is very different from an angiographic table, which is constructed to be transparent to $\mathrm{x}$-rays. Compared with a standard OR table, the angiography table has very few positioning options. However, the angiography table can be moved along its vertical axis to enable the imaging of multiple regions of the body during one examination without repositioning the patient. Such lengthwise mobility is limited in regular OR tables. For operations such as the cardiovascular interventions mentioned above, the longitudinal mobility of the table is not a compulsory requirement [10]. The patient is moved from the transport gurney and repositioned on the OR table outside the hybrid OR. The angiography table on the other hand is permanently fixed. Hence, the patient has to be repositioned in the angiography suite. Thus, the decision to install an OR table for a hybrid OR is easy to make if the OR is going to be used primarily for surgical purposes. With this hybrid design, however, a primarily interventional radiological use of the hybrid OR within the surgical unit is limited. The hybrid OR should be reserved for joint surgical-interventional procedures. In dedicated cardio-surgical centres the installation of a hybrid OR with an OR table is very useful if a full utilisation of the hybrid OR is guaranteed. An ideal direct connection of the heart catheter laboratory or the 
Fig. 1 In scenario 1, both stations are operated independently of each other. The connecting door remains closed. The left angiography station is equipped with a mobile C-arm. This is where venous interventions (implantation of port systems and tunnelled haemodialysis catheters) are performed.

The second angiography station is equipped with a bipolar angiography system

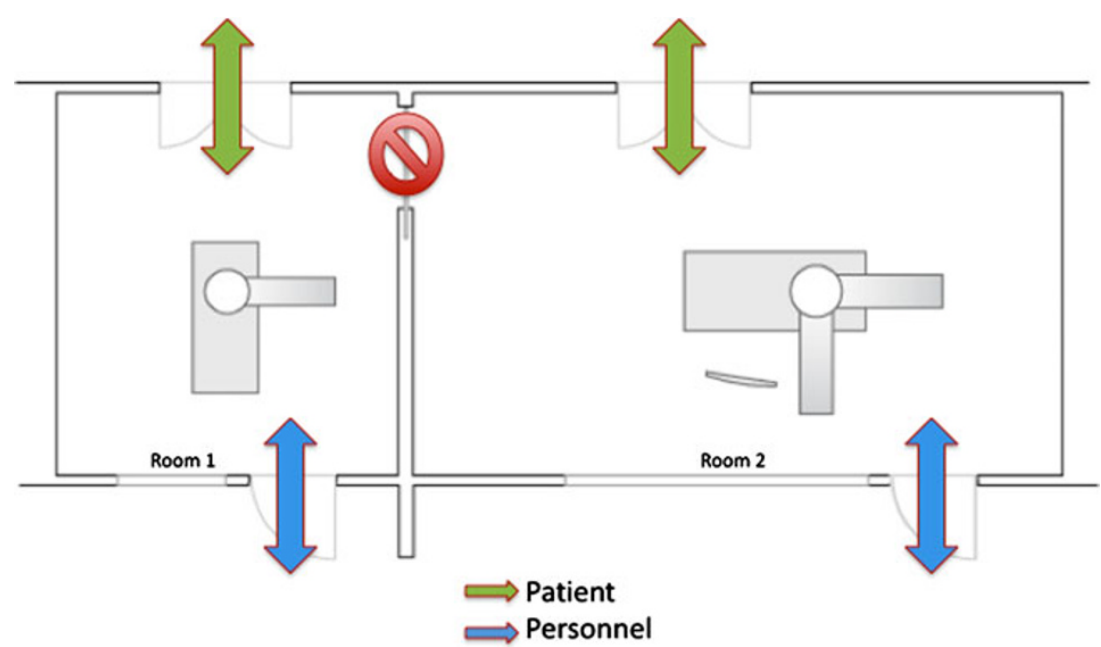

interventional radiological suite and the surgical wing is structurally impossible in most hospitals.

\section{Concept}

During the renovations of our angiographic-interventional suite, an alternative to the hybrid OR concept was implemented. Our institution cannot provide the case numbers required to justify an installation of an angiography system within the OR tract. However, we have noticed a steady increase in endovascular procedures, such as the interventional implantation of large lumen vessel prostheses, which should be implanted in an OR environment. With this concept, the additional option of utilisation for neurological interventions with a biplane angiography system and the further possibility of 3D rotation angiography is taken into account. The angiography system can be operated in a biplane mode during joint surgical-angiographic procedures, which are performed under full anaesthesia. An optional ultrasound device is also connected to the angiography system's monitors. On the other hand, we are seeing a marked increase in venous interventions (i.e., port implantations and tunnelled haemodialysis catheters) in interventional radiology. The requirements for venous interventions are typically the option of fluoroscopic imaging control of the catheter position. A modern DSA angiography system is not required.

To meet all of these demands, a two-room concept with an additional connecting door between the two rooms was chosen. The utilisation of the two rooms can be described in three scenarios: in scenario 1 both rooms are operated simultaneously (Fig. 1). The first room contains a mobile $\mathrm{C}$-arm for fluoroscopic imaging. Smaller venous interventions are performed here. The second room is equipped with a regular DSA angiographic system, which uses the biplane angiography unit. Both work stations are operated independently from one another.
Fig. 2 Scenario 2 describes a single room approach. The left intervention suite is used for preparations. The mobile $\mathrm{C}$-arm and the angiography table are moved aside. The workflow is optimised by opening the connecting door between the two suites. Direct interaction between the patients is precluded. While one patient is followed up, a second patient can be examined in the main angiography suite

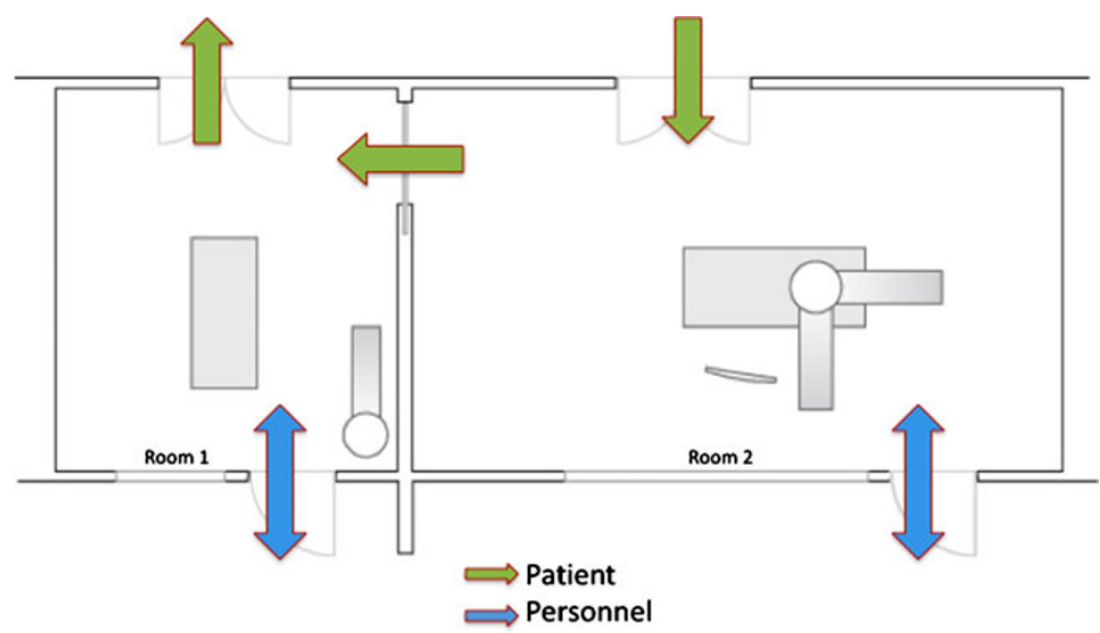


Fig. 3 Scenario 3 shows the hybrid use of both angiography suites. The left intervention suite is turned into a combined patient and staff hatchway. Direct access to the angiography suite is barred

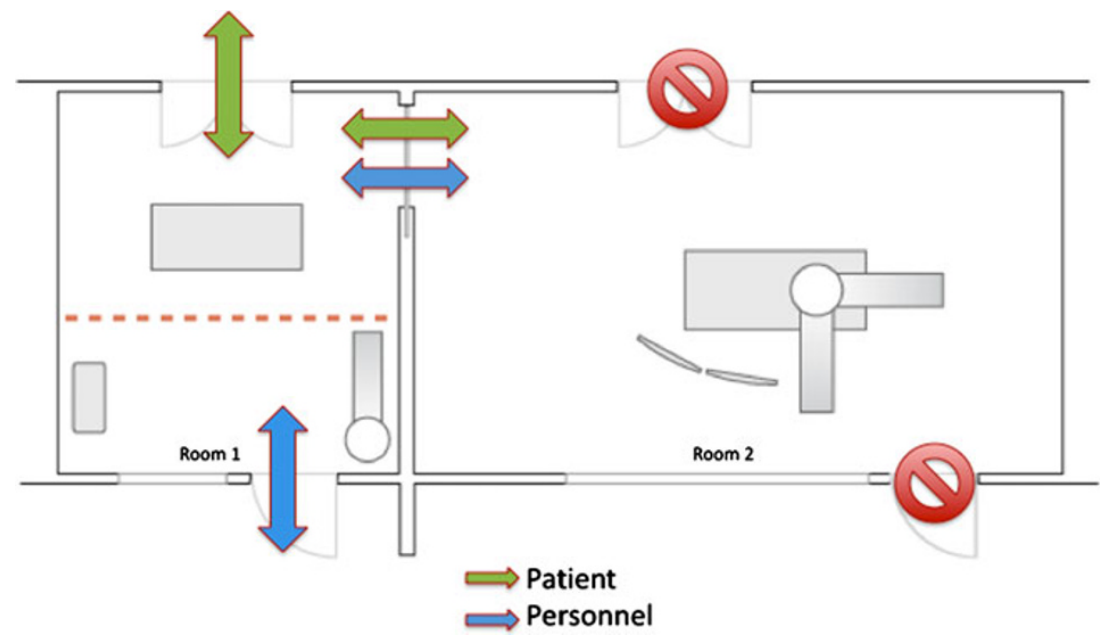

In scenario 2, the first angiography room is used for preparation and post-interventional patient care, such as manual compression of the puncture site, while the operating angiography room is prepared for the next patient. A connecting door between the two rooms provides an effective workflow in this one-room-utilisation concept (Fig. 2). While the first patient is taken care of post-intervention, the next patient is already being transferred into the angiography room.

The utilisation of the angiography suite as an angiographic OR is described in scenario 3 (Fig. 3). Direct access via the doors from the hallway and the console is barred during the use of the OR. The first room is used as a combined hatchway and OR preparation room in this scenario. Access to the angiography room is now only possible through the connecting door between the two rooms. The connecting door is a sliding OR door and can be operated automatically. The preparation room and the main angiography room are equipped with identical wall connections for medical gasses and patient monitoring.

The angiography suite was installed in close cooperation with our hospitals' hygienists to ensure that all requirements for the prevention of infections were complied with at organisational, functional-structural and technical levels. The national guidelines and recommendations on hygiene are compulsory and have to be implemented according to the local environment in detailed plans and instructions (standard operation procedures) in cooperation with the hospital's hygienists [11]. The OR should be a closed room with as few access points as possible. The OR should further have a personal hatchway that is detached from the rest of the hospital (Cat. IB) and should at least have a section for patient preparation (Cat. III) [11]. Both requirements are met in the concept we have introduced through a combined staff and patient hatchway.

This utilisation concept is designed primarily for interventional endovascular or percutaneous procedures. The abdominal cavity is only opened in case of an emergency. The room ventilation system was implemented without a laminar airflow (LAF) system. The angiography system interferes with the LAF to the point that a low turbulence suppression flux cannot be achieved. The same is true when additional devices, such as a C-arm, are brought into a regular OR. An LAF ventilation system is only required for aseptic procedures with a particularly high risk of infection [12-16]. Such high-risk procedures are not planned in our utilisation concept. If a serious adverse event occurs in the angiosuite (e.g., conversion to an open AAA repair), the open operation is done directly on the angiography table in the angiosuite. It should be noted at this point that from a hygiene point of view LAF cannot be warranted in any room concept if an x-ray device is used. In December 2009, the German Commission for the Prevention of Infection and Hospital Hygiene downgraded LAF ventilation systems to the category of "unresolved questions". Thus, it is likely that in the future even procedures with an increased risk of infection (e.g. implantation of hip prostheses) will not require LAF.

\section{Conclusion}

With this angiographic-interventional suite design, a flexible demand-orientated adaptation of the utilisation concept to every need of modern intervention radiology is possible. The increasing challenges of interdisciplinary cooperation-especially with vascular surgery-are met with this concept. Endovascular interventions with a primary focus on aortic stent grafts are performed in a technically and therapeutically ideal environment. The professional cooperation between vascular surgery and interventional radiology in the angiographic OR intensifies and improves the relationship between the two specialties, turning the theoretical approach of an interdisciplinary centre for vascular interventions into a vital organism. 


\section{References}

1. Uflacker R, Robison J (2001) Endovascular treatment of abdominal aortic aneurysms: a review. Eur Radiol 11:739-753

2. Krauss T, Pfammatter T, Mayer D, Lachat M, Hechelhammer L, Marincek B, Frauenfelder T (2010) Hybrid-repair of thoracoabdominal or juxtarenal aortic aneurysm: what the radiologist should know. Eur Radiol 20:1011-1022

3. Steingruber IE, Czermak BV, Chemelli A, Glodny B, Bonatti J, Jaschke W, Waldenberger P, Rieger M, Neuhauser B (2007) Placement of endovascular stent-grafts for emergency repair of acute traumatic aortic rupture: a single-centre experience. Eur Radiol 17:1727-1737

4. Thurnher SA, Grabenwöger M (2002) Endovascular treatment of thoracic aortic aneurysms: a review. Eur Radiol 12:1370-1387

5. Duda SH, Pusich B, Raygrotzki S, Uckmann FP, Aebert H, Tepe G (2002) Endovascular implantation of stent-grafts in the thoracic aorta-mid-term results of a prospective controlled study. Rofo 174:485-489

6. Bonatti J, Vassiliades T, Nifong W, Jakob H, Erbel R, Fosse E (2007) How to build a cath-lab operating room. Heart Surg Forum 10:344-348

7. Kiaii B, McClure RS, Kostuk WJ, Rayman R, Swinamer S, Dobkowski WB, Novick RJ (2005) Concurrent robotic hybrid revascularization using an enhanced operative suite. Chest 128:4046-4048

8. Slovut DP, Bacharach JM (2004) Aortic aneurysm repair with endovascular grafts: developing a graft surveillance program. Catheter Cardiovasc Interv 62:252-261
9. Willoughby RP, Fenton JA, Pudupakkam SR, Greco RA, Roberts EW, DeRose G, Kribs S (2004) Implementation of a successful endovascular surgical program in a non-teaching tertiary-care centre in Ontario. Can J Surg 47:182-188

10. ten Cate G, Fosse E, Hol PK, Samset E, Bock RW, McKinsey JF (2004) Integrating surgery and radiology in one suite: a multicenter study. J Vasc Surg 40:494-499

11. Anforderungen der Hygiene bei Operationen und anderen invasiven Eingriffen (2000) Bundesgesundheitsbl-GesundheitsforschungGesundheitsschutz. 43:64-648

12. Brandt C, Hott U, Sohr D, Daschner F, Gastmeier P, Rüden H (2008) Operating room ventilation with laminar airflow shows no protective effect on the surgical site infection rate in orthopedic and abdominal surgery. Ann Surg 248:695-700

13. Mangram AJ, Horan TC, Pearson ML, Silver LC, Jarvis WR (1999) Guideline for prevention of surgical site infection, 1999. Centers for Disease Control and Prevention (CDC) hospital infection control practices advisory Committee. Am J Infect Control 27:97-132

14. Geffers C, Gastmeier P, Daschner F, Rüden H (2001) Prevention of postoperative wound infections. Evidence-based recommendations. Zentralbl Chir 126:84-92

15. Lidwell OM (1986) Clean air at operation and subsequent sepsis in the joint. Clin Orthop Relat Res 211:91-102

16. Ritter MA, Stringer EA (1980) Laminar air-flow versus conventional air operating systems: a 7-year patient follow-up. Clin Orthop Relat Res 150:177-180 